\title{
Neuroplastic changes in patients with schizophrenia undergoing cognitive remediation: triple-blind trial
}

\author{
Ian S. Ramsay, Tasha M. Nienow, Matthew P. Marggraf and Angus W. MacDonald
}

\section{Background}

Patients with schizophrenia have shown cognitive improvements following cognitive remediation, but the neuroplastic changes that support these processes are not fully understood.

\begin{abstract}
Aims
To use a triple-blind, placebo-controlled trial to examine neural activation before and after cognitive remediation or a computer skills training (CST) placebo (trial registration: NCT00995553)).

\section{Method}

Twenty-seven participants underwent functional magnetic resonance imaging before and after being randomised to either cognitive remediation intervention or CST. Participants completed two variants of the N-back task during scanning and were assessed on measures of cognition, functional capacity, community functioning and symptoms.
\end{abstract}

\section{Results}

We observed a group $\times$ time interaction in the left prefrontal cortex, wherein the cognitive remediation group showed increased activation. These changes correlated with improved task accuracy within the cognitive remediation group, whereas there was no relationship between changes in activation in untrained cognitive measures. Significant changes were not observed in other hypothesised areas for the cognitive remediation group.

\section{Conclusions}

We replicated the finding that cognitive remediation increases left lateral prefrontal activation during a working memory task in patients with schizophrenia, suggesting this may be an important neural target for these types of interventions.

\section{Declaration of interest}

None.

\section{Copyright and usage}

(c) The Royal College of Psychiatrists 2017.
Cognitive remediation is becoming an increasingly promising class of interventions to alleviate the cognitive deficits associated with schizophrenia and related illnesses. Although emerging evidence suggests that therapist-guided computerised training can improve both cognition and functioning in schizophrenia, ${ }^{1,2}$ we are only just beginning to identify the neural substrates that support this change. In a meta-analysis of studies examining changes in brain activity following cognitive remediation for schizophrenia, areas of both the prefrontal and subcortex showed increases following treatment. ${ }^{3}$ Identifying the brain regions that both respond to and reflect cognitive changes from cognitive remediation will be critical as we begin to specifically target impaired cognitive domains by exercising their disrupted constituent neural systems. Working memory is one such target system that is both impaired in schizophrenia and associated with prefrontal disruption. ${ }^{4}$ These disruptions are thought to affect other impaired domains of cognition and functioning, ${ }^{5}$ making working memory a critical behavioural target for cognitive remediation interventions. Previous work in our own laboratory found evidence of activation change (neuroplasticity) in prefrontal cortical activation patterns during a working memory task following working memory-focused cognitive remediation. Patients with schizophrenia randomised to undergo up to $25 \mathrm{~h}$ of cognitive remediation (compared with a cognitive-behavioural social skills training control condition $)^{6}$ showed prefrontal activation increases and associated improvements in performance on working memory tasks. ${ }^{7}$ To expand on these promising preliminary results, the current study aimed to replicate the activation findings of Haut and colleagues, ${ }^{7}$ by examining a subgroup of participants from a double-blind, active placebocontrolled study, who received a working memory-focused cognitive remediation protocol for schizophrenia. We also extended this inquiry to examine relationships between neural changes and cognitive, psychosocial functioning and symptom outcomes associated with training. We hypothesised that changes in functional activation from cognitive remediation would coincide with changes in cognition and functioning. Last, we sought to determine whether patients' baseline cognition, functioning or symptom profile was predictive of neural response to training. Based on previous behavioural findings, ${ }^{8-10}$ we hypothesised that higher baseline functioning and cognition would predict a greater neuroplastic response to training.

\section{Methods}

\section{Participants}

Patients with schizophrenia or schizoaffective disorder who had previously elected to participate in a clinical trial of working memory-focused cognitive remediation (trial registration: NCT00995553) were recruited for the current study. Diagnosis was established using the Structured Clinical Interview for DSM-IV Axis I Disorders. ${ }^{11}$ Master's and doctoral-level clinicians conducted the interviews (mean kappa scores ranged from $0.87-$ 1.00 across all diagnostic categories, and $0.92-1.00$ for psychotic disorders), and the principal investigator (T.M.N.) assigned a study diagnosis after review of available medical records, patient reports and interviewer observations. All participants were between 18 and 60 years old; were clinically stable, with no antipsychotic medication changes or admissions to hospital in the previous month; had a Wechsler Test of Adult Reading $(\text { WTAR })^{12}$ score of 70 or above; had no substance/alcohol 
dependence within the past 6 months and no substance/alcohol misuse in the past month; had no history of head injury with loss of consciousness for greater than $20 \mathrm{~min}$; and had no known history of neurological conditions that could comprise cognitive functioning. All participants demonstrated capacity to give consent.

In total, 53 of the 85 participants enrolled in the clinical trial were screened for magnetic resonance imaging (MRI) compatibility (funding for the imaging protocol was secured after 32 participants had already been randomised and started training) and 40 consented to the imaging study. Three participants were withdrawn prior to scanning after additional review of their medical history found them to be ineligible. Two additional participants were withdrawn because of inability to complete the scans. Five participants chose to withdraw because of lack of interest $(n=3)$ or anxiety in the scanner $(n=2)$. A total of 30 participants completed scanning but data from 3 were not useable because of experimenter error; 27 participants completed all study procedures. No participants were removed after employing a $2 \mathrm{~mm}$ mean displacement movement cut-off within each scan. The protocol and consent process were approved by the Institutional Review Board at both the University of Minnesota and the Minneapolis VA Health Care System (VAHCS).

\section{Study design and masking}

Participants were randomised to undergo either a working memory-focused cognitive remediation intervention (the cognitive remediation group, $n=15$ ) or an active computer skills training (CST) control (the CST group, $n=12$ ). Although 15 participants underwent cognitive remediation, one did not complete the picture $\mathrm{N}$-back at both time points, leaving $n=14$ in the cognitive remediation group for that task. The same therapists provided both kinds of interventions and understood both arms to be active interventions. Assessments were conducted masked to group membership, and functional MRI (fMRI) analyses were conducted using de-identified groups. This final mask was not broken until whole region of interest (ROI) confirmatory analyses were completed as described below.

\section{Training procedure}

All training took place at the Minneapolis VAHCS as part of a larger trial examining cognitive and behavioural outcomes associated with a working memory-focused cognitive remediation. Participants completed $48 \mathrm{~h}$ of training over the course of 16 weeks (typically, three $1 \mathrm{~h}$ sessions weekly) in either the cognitive remediation or CST group. Groups did not statistically differ with regard to average number of training hours (cognitive remediation group 48.00, s.d. =0.0; CST group 47.91, s.d. $=0.28$ ).

Participants randomised to the cognitive remediation group completed a computer-based training program that consisted of 21 adaptive computer exercises that placed demands on working memory through verbal, visual and spatial stimulus modalities (online Table DS1). The tasks were selected from the Psychological Software Services CogRehab program (Psychological Software Services, Indianapolis, 2009), BrainTrain's educational software (Capitan's Log, 2010) and variants of N-back tasks using English words and other stimuli. ${ }^{13}$ Approximately a third of training time focused specifically on training with a version of the N-back task (0, 2, 3 or 4-back). Participants were advanced to a higher training level after demonstrating mastery performance ( $85 \%$ accuracy) at the previous level across three consecutive administrations of the task.
Participants randomised to the active placebo control group engaged in a CST course, focusing on keyboard skills and learning to use Microsoft Office for word processing, spreadsheet management and presentation creation. The CST condition was designed to have the same level of training time, exposure to computers and attention from treatment providers as the cognitive remediation condition.

Both groups were facilitated by Master's or Bachelor's level interventionists, who provided instruction, monitored progress, acknowledged effort and intervened as necessary to minimise frustration. Interventionists and participants were told that the study was an examination of how two types of skills training had an impact on functioning in the community. Additionally, a doctoral-level clinician (T.M.N.) led weekly $30 \mathrm{~min}$ bridging groups, where members processed their reactions to the training, and discussed skills they were learning and how they could be applied in real-life situations.

\section{Assessment procedure}

Enrolled participants underwent clinical, cognitive and functional assessment at baseline and after 4 months of training. Cognitive assessment included two putatively neutral variants of the N-back task: an English word version and a picture version featuring still-frame images of animals. Both tasks switched between blocks of 2-back and 0-back trials (online Fig. DS1). Five variants of the word N-back task, including the version used at the pre- and postintervention assessment, were practised throughout the training procedure in the cognitive remediation group. Participants also spent an equal amount of time training on seven variants of the picture N-back task. A picture N-back task with novel stimuli was included to measure skill transfer and generalisation (i.e. improving performance to untrained stimuli) to an untrained version of the working memory task. This assessment was conducted at baseline and post-intervention both in and out of the scanner. N-back performance was assessed using the sensitivity measure D-prime $\left(\mathrm{D}^{\prime}\right)$, which compared the number of 'hits' to 'false alarms' in the 2-back condition. ${ }^{14}$

Other cognitive measures included the MATRICS Consensus Cognitive Battery (MCCB), ${ }^{15}$ which was specifically developed to assess cognitive functioning in schizophrenia, and measures domains of attention/vigilance, speed of processing, working memory, verbal learning, visual learning, problem-solving/ reasoning and social cognition. For this study, we specifically examined the working memory, attention/vigilance and overall cognitive functioning domains as dependent variables to measure near transfer (generalisation to untrained tasks within the same domain of cognition) and far transfer (generalisation to unrelated skills with distinct task demands) in the context of our working memory-focused cognitive remediation intervention.

Functional capacity was measured using the UCSD Performance-Based Skills Assessment (UPSA) overall score that measures skilfulness on activities of daily living. ${ }^{16}$ Capacity for social competence was measured with the Social Skills Performance Assessment (SSPA) overall score, which rates domains of social competence, including appropriateness, clarity, fluency, affect, interest/disinterest, grooming and overall conversation skills based on role-plays of social interactions with a confederate. ${ }^{17}$ Clinical raters achieved mean interclass correlation coefficients (ICC) of 0.82 to 0.95 on the SSPA total score and 0.70 to 0.80 on the subscale scores. The total score from the self-report Social Functioning Scale (SFS; seven subscales assessing frequency of engagement in social and recreational activities, independent living skills and employment) was used 
to examine level of functioning in the community ${ }^{18}$ ICCs for the SFS were 0.88 or higher for both the total and subscale scores.

Clinical symptoms were assessed using the expanded Brief Psychiatric Rating Scale (BPRS), ${ }^{19}$ a 24 -item inventory that measures severity of positive symptoms, negative symptoms, depression, mania and disorientation. For the current study we relied on the total score as a measure of psychiatric symptomology. Mean ICCs on BPRS items ranged from 0.80 to 0.87 .

\section{Imaging procedure}

Scanning took place within a week of the class beginning or ending. In the scanner, participants completed the two previously described variants of the N-back (with condition order counterbalanced). Both versions randomly switched between 0-back trials and 2-back trials to measure baseline encoding and working memory functioning, respectively. Items were displayed on the screen for $500 \mathrm{~ms}$ with an interstimulus interval of $2000 \mathrm{~ms}$. Each task pseudo-randomly switched between blocks of 0-back and 2-back trials. All participants briefly practised the N-back tasks outside of the scanner before each imaging session to ensure understanding of the task demands. The procedure including consenting, training, scanning and debriefing took approximately $1.5 \mathrm{~h}$.

The fMRI scans were conducted at the University of Minnesota Center for Magnetic Resonance Research. Participants were counterbalanced to receive either the word or picture N-back first. Each $\mathrm{N}$-back was performed over two scanning blocks, each containing 212 functional scans, for a total of 424 functional scans per task. Images were collected using a 3-Tesla Siemens Trio MRI scanner, and a 32-channel head coil (repeat time $(\mathrm{TR})=1.5 \mathrm{~s}$, echo time $(\mathrm{TE})=40$, flip angle $90^{\circ}$, voxel size $3.4 \times 3.4 \times 5 \mathrm{~mm}$ thickness, field of view $(\mathrm{FOV})=22 \mathrm{~cm}, 35$ axial slices $). T_{1}$ reference images were also collected (voxel size $=1 \times 1 \times 1.2 \mathrm{~mm}$ thickness, $240 \times 256 \times 160$ dimensions). Data were preprocessed using FSL (http://www.fmrib.ox.ac.uk/fsl/). Images were spatially normalised in a two-step procedure using rigid body transformations (FLIRT), in which the images were first normalised to the individual structural image, and then to the standard template. Field maps were collected to carry out B0-unwarping, and motion correction used rigid body transformations (MCFLIRT) with a six-parameter motion regression procedure. Scans were spatially smoothed at full-width at half maximum (FWHM) $=7 \mathrm{~mm}$, normalised using the mean volume intensity and filtered with a high pass frequency cut-off of $100 \mathrm{~s}$. All models included temporal derivatives. Mean average displacement (movement) across all scan sessions was $0.24 \mathrm{~mm}$ (s.d. $=0.30$ ).

\section{Planned analyses}

Behavioural analyses of the two N-back tasks to measure training gain and generalisation were examined by entering $\mathrm{d}^{\prime}$ scores for the 2-back condition in a repeated-measures ANOVA. Paired-sample $t$-tests were used to explore effects.

Functional imaging analyses were conducted in FSL using a general linear model (GLM) procedure, wherein participants' fMRI time series were modelled in a block design comparing 2-back with 0-back blocks ( $2 \mathrm{Bv} 0 \mathrm{~B})$ for each task. Confirmatory imaging analyses relied on five ROIs previously shown to improve with cognitive remediation, ${ }^{7}$ four of which showed greater increases in activity among patients with performance improvements on an N-back task. The peak voxels of these previously observed regions were dilated to create spherical ROIs approximating a similar volume for each individual region (online Fig. DS2). We first performed a confirmatory voxel-wise analysis constrained to the same five ROIs in a small-volume correction. Group images were cluster-thresholded within the ROI at $Z>2.3$ and a significance threshold of $P=0.05$. Next, individual participant's beta values ( $\%$ signal change) were extracted for each of the five ROIs individually and entered into a repeated-measures ANOVA to examine group $\times$ time interactions in the statistical program R.

After confirmatory analyses were completed, the masking was broken. Next, we performed correlations between change in activation observed in the voxel-wise analyses in the cognitive remediation group (individual participant \% signal change was extracted and averaged across the voxels surviving correction), with the previously described outcome measures of cognition, functioning and symptoms. All tests were calculated one-tailed, as we hypothesised based on previous findings ${ }^{7,20}$ that increased activation would correspond with improved cognition and outcome. Finally, we examined whether baseline measures of cognition, functioning and symptoms correlated with activation change observed in the voxels previously described.

\section{Results}

\section{Sample characteristics}

Individuals in the imaging study did not differ from those in the larger behavioural trial on the basis of gender, age, ethnicity, education, parental education or chlorpromazine-equivalents medication dosage (all $P s>0.13$ ). In addition, participants randomised to the two treatment groups did not differ on demographic, clinical, cognitive or functional measures at baseline (Table 1).

\section{Behavioural treatment effects measured by the $\mathrm{N}$-back}

We first examined whether the intervention influenced working memory performance on the N-back tasks. $\mathrm{D}^{\prime}$ values from the 2-back conditions were entered in repeated-measures ANOVAs. The word N-back did not show a group $\times$ time interaction, but did show a significant effect of time across groups $\left(F(1,25)=10.57, P<0.005, \eta^{2}=0.30\right)$, with increases in $\mathrm{D}^{\prime}$ from pre- to post-training for both the cognitive remediation $(t=2.25(14), P<0.05, d=0.41)$ and CST $(t=2.40(11), P<0.05$, $d=0.59$ ) groups (online Fig. DS2(b)). $\mathrm{D}^{\prime}$ values on the picture $\mathrm{N}$-back showed a trend for a group $\times$ time interaction $\left(F(1,24)=3.63, P=0.07, \eta^{2}=0.13\right)$, wherein participants in the cognitive remediation group improved performance from pre(mean 2.62, s.d. $=0.60$ ) to post-training (mean 3.15, s.d. $=0.82$; $t=2.25(12), P<0.05, d=0.74$ ), whereas the CST group showed no significant change over time (Fig. 1). Because the behavioural results from the picture N-back appeared to be more sensitive to the effects of the intervention, imaging analyses focused on that task.

\section{Voxel-wise and individual ROI confirmatory imaging results}

Confirmatory analyses examined the average $2 \mathrm{Bv} 0 \mathrm{~B}$ contrast activations in five ROIs identified by Haut and colleagues ${ }^{7}$ in a previous examination of cognitive remediation in schizophrenia (online Fig. DS2(a)). First, to examine voxel-wise activation changes in these areas, we conducted a small volume analysis using the previously defined ROIs as a mask. We observed a group $\times$ time interaction in a subset of the left dorsolateral prefrontal cortex (DLPFC), wherein activation in the cognitive remediation group increased from pre- to post-training, but not 


\begin{tabular}{|c|c|c|c|c|}
\hline & $\begin{array}{l}\text { Cognitive remediation group, } \\
\text { mean (s.d.) }(n=15)\end{array}$ & $\begin{array}{l}\text { Computer skills training group, } \\
\text { mean (s.d.) }(n=12)\end{array}$ & $t$ & $P$ \\
\hline Age, years & $42.93(10.60)$ & $45.75(7.70)$ & 0.80 & 0.43 \\
\hline Education, years & $13.47(1.50)$ & $12.42(1.04)$ & 1.20 & 0.24 \\
\hline Parental education, years & $12.83(4.30)$ & $13.21(1.79)$ & 0.30 & 0.76 \\
\hline Wechsler Test of Adult Reading IQ, standard score & $104(10.76)$ & $101.42(11.56)$ & 0.60 & 0.56 \\
\hline Duration of illness, years & $20.93(12.73)$ & $18.5(11.11)$ & 0.53 & 0.60 \\
\hline Total chlorpromazine equivalents & $551.80(466.24)$ & $320.75(280.81)$ & 1.60 & 0.12 \\
\hline \multicolumn{5}{|l|}{ Baseline scores } \\
\hline Word N-back (D') & $2.74(0.90)$ & $2.32(0.70)$ & 1.34 & 0.19 \\
\hline Picture N-back (D') & $2.54(0.64)$ & $2.54(0.88)$ & 0.00 & 0.99 \\
\hline \multicolumn{5}{|l|}{ MATRICS Consensus Cognitive Battery, T score } \\
\hline Working memory & $42.33(12.19)$ & $43.33(15.98)$ & 0.18 & 0.86 \\
\hline Attention & $43.27(10.23)$ & $40.08(10.51)$ & 0.79 & 0.44 \\
\hline Overall & $37.00(16.34)$ & $34.00(15.27)$ & 0.49 & 0.63 \\
\hline UCSD Performance-Based Skills Assessment, total score & $74.00(12.25)$ & $73.67(16.15)$ & 0.06 & 0.95 \\
\hline Social Skills Performance Assessment, total score & $70.20(10.20)$ & $63.08(12.81)$ & 1.57 & 0.13 \\
\hline Social Functioning Scale, total score & $122.47(14.09)$ & $116.42(12.80)$ & 1.17 & 0.25 \\
\hline Brief Psychotic Rating Scale, total score & $42.53(9.74)$ & $45.00(11.17)$ & 0.60 & 0.55 \\
\hline
\end{tabular}

the CST group (Fig. 2(a) and (b); Table 2). Mean activation changes of the voxels in the left DLPFC (post $>$ pre) correlated with change in $\mathrm{D}^{\prime}$ score on the picture N-back task $(r=0.51$, $P($ one-tailed $)<0.05) \quad$ (Fig. 2(c)). No activation changes were observed in other ROIs, and no changes were observed at the whole brain level (uncorrected whole brain results at $P<0.005$ can be found in online Table DS3 and Fig. DS3).

Next we conducted confirmatory analyses of each ROI individually by extracting mean \% signal change values from the whole ROI and entering them into a repeated-measures ANOVA. No whole individual ROI showed a significant group $x$ time interaction. However, the left DLPFC ROI showed a trending interaction favouring the cognitive remediation group $(F(1,24)=$ $\left.3.16 p=0.09, \eta^{2}=0.11\right)$, supported by an increase in activation in the cognitive remediation group from pre to post-training $(t=2.46$ (13), $P$ (one-tailed $)<0.05, d=0.53)$. Additionally, the left frontal pole ROI showed a significant effect of time $\left(F(1,24)=10.17, P<0.005, \eta^{2}=0.28\right)$, with an unexpected decrease in activation from pre- to post- training in the CST group

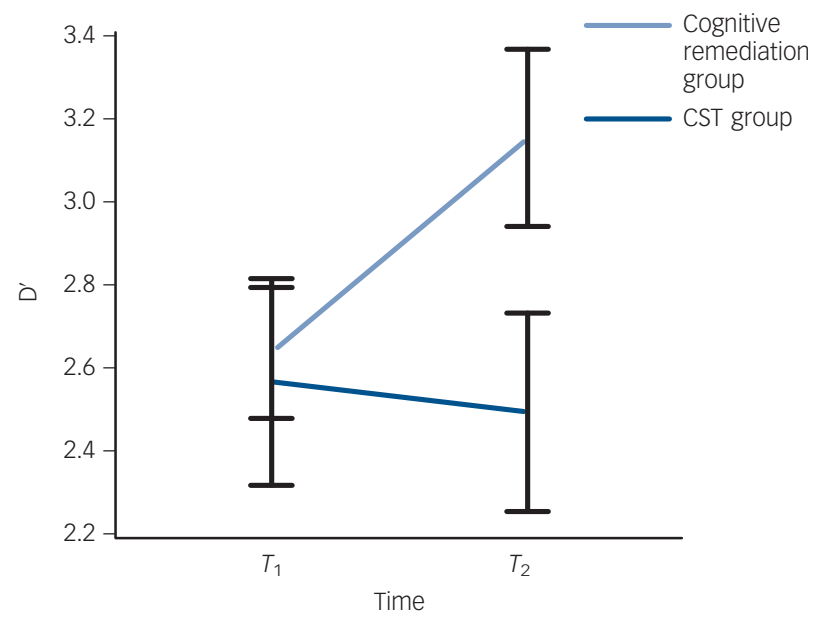

Fig. 1 Picture N-Back task behavioural findings.

Patients in the cognitive remediation intervention group showed an increase in $\mathrm{d}^{\prime}$ from time $1\left(T_{1}\right.$, mean 2.62 , s.d. $\left.=0.60\right)$ to time $2\left(T_{2}\right.$, mean 3.15 , s.d. $\left.=0.82\right)$ on the 2 -back trials of the picture $\mathrm{N}$-back task (results using \%-correct can be found in online Table DS2), whereas those in the computer skills training group (CST group) showed no change from $T_{1}$ (mean 2.54 , s.d. $=0.88$ ) to $T_{2}$ (mean 2.47, s.d. $=0.86$ ). $(t=-2.93(11), \quad P<0.05, \quad d=1.31)$. Hypothesised changes in ROI activation for the cognitive remediation group did not significantly correlate with changes in N-back performance.

\section{Change in DLPFC on 2Bv0B predicting change in outcomes}

We examined whether change in activation observed in the cognitive remediation group during $2 \mathrm{Bv} 0 \mathrm{~B}$ was related to changes in cognition. Although changes in left DLPFC activation correlated with improvements in $\mathrm{D}^{\prime}$ on the N-back task, this pattern was not observed in the hypothesised near transfer working memory or attention/vigilance domains from the MCCB. Change in left DLPFC activation also did not relate to change in the MCCB overall composite score.

Additionally, we examined whether activation change in the cognitive remediation group related to functional and symptom change. Left DLPFC activation change did not correlate with changes in SSPA or SFS performance, although no significant behavioural changes were observed on these measures. A nonsignificant trend in the hypothesised direction $(r=0.42$, $P$ (onetailed $)=0.07$ ) was found between change on the UPSA and left DLPFC activation. Change in BPRS total score was also not related to change in functional activation.

\section{Baseline cognition and behaviour predicting functional activation change}

Finally, we examined whether baseline measures of cognition, functional capacity, community functioning or symptoms were predictive of plasticity in the left DLPFC for the cognitive remediation group. No measure of interest, including baseline N-back performance, MCCB scores, SSPA, SFS, UPSA or BPRS were predictive of activation increases in the left DLPFC during the 2Bv0B contrast (all $P_{\mathrm{s}}>0.11$ ).

\section{Discussion}

\section{Main findings and comparison with findings from previous studies}

The current study examined the neural response to a working memory-focused cognitive remediation intervention in schizophrenia and explored the clinical significance of observed brain 
(a)

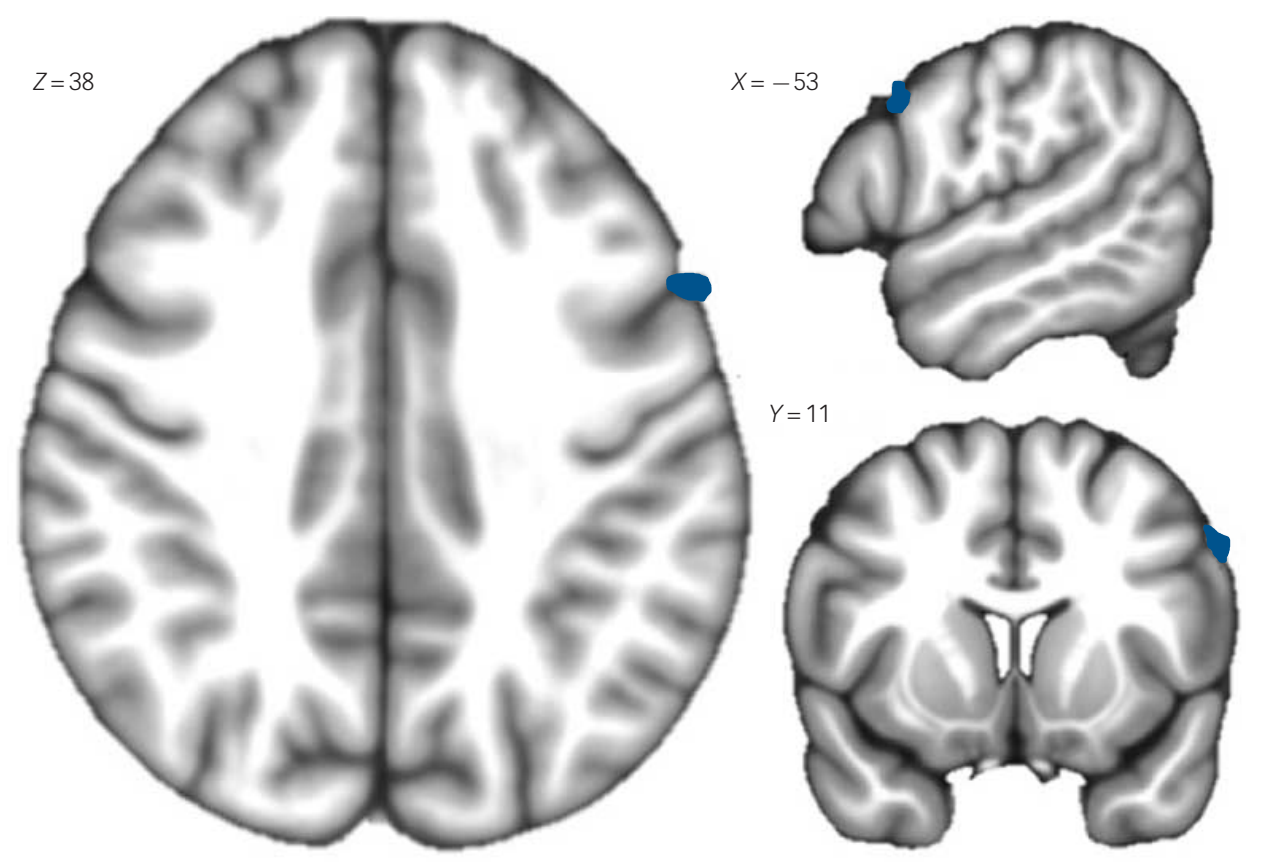

(b)

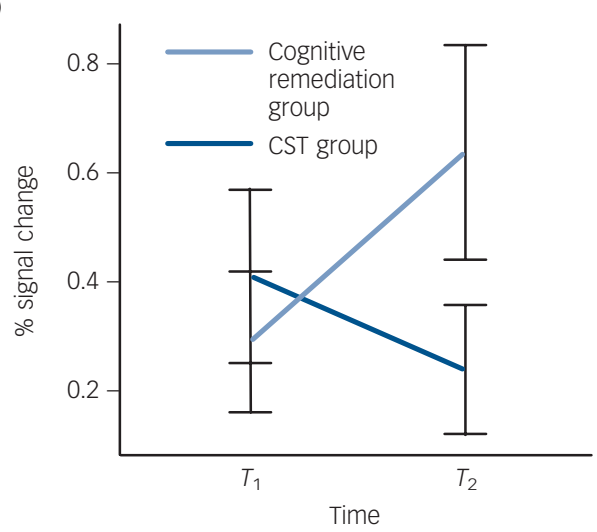

(c)

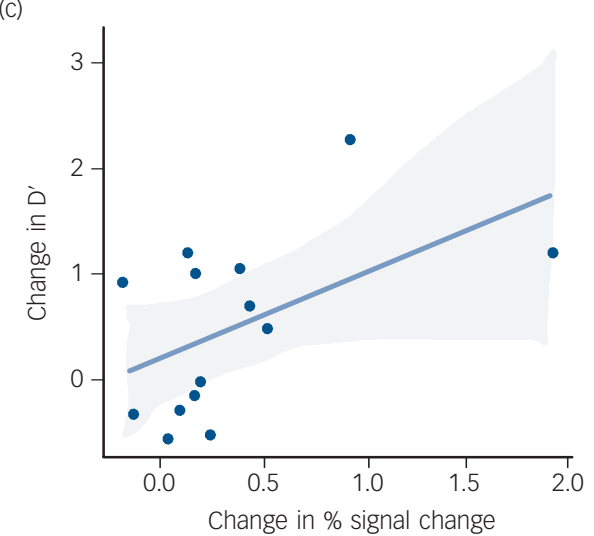

Fig. 2 Group $\times$ time interaction in the left dorsolateral prefrontal cortex (DLPFC) region of interest (ROI) for 2-back v. 0-back blocks (2Bv0B).

We observed a voxel-wise group $\times$ time interaction in the left DLPFC ROI during the $2 \mathrm{BVOB}$ condition in the cognitive remediation intervention group (a) driven by increases from pre- to post-training in the cognitive remediation group but not the computer skills training group (CST group) (b). (c) Change in per cent signal change extracted from the significant voxels in the left DLPFC were positively correlated with changes in $d^{\prime}$ on the picture $\mathrm{N}$-back task $(r=0.51, P$ (hypothesised) $<0.05)$. This relationship held when using a robust linear estimator to control for the effects of outliers $(r=0.56, P($ hypothesised $)<0.05)$.

changes. In doing so, we sought to expand on previous findings that showed functional activation changes in prefrontal areas supporting improvements on a working memory task. ${ }^{7}$ Consistent with hypotheses, we observed a group $\times$ time interaction in a subgroup of voxels in the left DLPFC, wherein the cognitive remediation group increased activation following training. Changes in these voxels correlated with improvements on the picture N-back task in the cognitive remediation group. We also observed a marginal trend showing that change in DLPFC activation was related to change in functional skill performance measured by the UPSA. However, we did not show transfer to other measures of working memory, attention, global cognition, social competence or community functioning.

Contrary to expectations, we did not observe confirmatory functional activation change in the other four hypothesised ROIs during the $2 \mathrm{Bv} 0 \mathrm{~B}$ contrast. This indicates that the current cognitive remediation intervention either did not sufficiently invoke plasticity in these regions, or that we were underpowered to demonstrate such change. Overall, the results of this study indicate that cognitive remediation has less neuroplastic influence and generalisability than found by Haut et al. ${ }^{7}$ However, our replication of activation in the left DLPFC reinforces its importance in supporting N-back generated working memory performance and suggests that this region could serve as a more focal target (i.e. DLPFC) for future intervention efforts.

Also unexpected was our failure to observe a significant group $\times$ time interaction on the word N-back task. It is unclear why both groups improved on this task while the expected postintervention between-group difference was observed on the picture N-back. However, it is noteworthy that both groups showed strong performance at baseline across tasks, potentially limiting our ability to detect between-group differences in task improvement. The verbal nature of the word N-back task might have been easier to encode, resulting in performance improving quickly for all participants once they were familiar with the task. In our previous study, ${ }^{7}$ untrained healthy controls improved on this task between baseline and post-intervention assessment, suggesting that this task might be more susceptible to this type 


\begin{tabular}{|c|c|c|c|c|c|}
\hline Region & Voxels, $n$ & Z-Max & $x$ & $y$ & $z$ \\
\hline Left middle frontal gyrus & 19 & 3.52 & -56 & 12 & 38 \\
\hline Left precentral gyrus & & 2.69 & -56 & 12 & 32 \\
\hline
\end{tabular}

of practise effect. Alternatively, CST might also have had an influence on cognition and neural plasticity. In a similar study of cognitive remediation with a computer-based control condition, performance improved across groups on some working memory tasks. ${ }^{21}$ However, we did not observe significant relationships between word N-back behavioural performance and cognition, functioning, symptoms or functional activation changes. Therefore, the significance of this change remains unclear, but may be most likely because of practise effects.

Our finding that change in activation in a subset of the DLPFC during $2 \mathrm{Bv} 0 \mathrm{~B}$ on the picture N-back correlated with change in picture N-back task performance is consistent with previous cognitive remediation studies in schizophrenia that have found neural change in response to training and that the magnitude of change was related to behavioural task performance. For instance, Subramaniam and colleagues examined a letter $\mathrm{N}$-back task before and after undergoing 16 weeks of an auditory training intervention and showed increased ROI activation in the left middle and inferior frontal gyrus, as well as bilateral insula. ${ }^{22}$ Additionally, changes in the right middle frontal gyrus activation were positively correlated with changes in working memory performance on the 2-back task. Similarly, Bor and colleagues used a cognitive remediation intervention that targeted attention, working memory, logical thinking and problem-solving over a 7-week training period. They demonstrated that cognitive remediation increased activation on a spatial N-back task in the left inferior and middle frontal gyrus, cingulate gyrus and precuneus. ${ }^{23}$ Additionally, Haut and colleagues had congruent findings with a protocol quite similar to the current study. ${ }^{7}$ Taken together, observations from these studies suggest that cognitive remediation may have the capacity to increase prefrontal activation in brain areas previously shown to be disrupted in schizophrenia. $^{24}$

This study is one of a few imaging protocols to examine whether activation change in response to a discrete task also correlated with change in clinically meaningful targets. Although activation change was not related to change in measures of cognition, social competence, community functioning or symptomology, there was a trend, albeit uncorrected, with improvements in functional capacity measured by the UPSA. Although this needs to be interpreted cautiously, this finding may indicate that working memory training for schizophrenia may support aspects of recovery by improving brain function. This coincides with previous findings demonstrating that changes in prefrontal activation were predictive of occupational functioning 6 months following cognitive training. ${ }^{22}$ Behavioural studies have indicated that neuropsychological measures strongly predict functional capacity in schizophrenia, ${ }^{25,26}$ and the current findings suggest that working memory training may have an impact on this relationship. However, this will also require replication in larger samples to better understand whether these tentative relationships are truly meaningful.

We did not observe relationships between baseline cognitive, psychosocial or symptom measures and neuroplastic changes associated with cognitive remediation. Two studies have demonstrated that baseline working memory and other neuropsychological measures can be predictive of the course of treatment and response to cognitive remediation. ${ }^{8,27}$ We sought to extend this work by examining whether individual differences before treatment were predictive of neural changes. Although no significant relationships were found, it is important to note that the current study was not powered to be sensitive to detect predictors with a small effect. However, our findings are consistent with meta-analytic findings that also show no consistent pre-treatment characteristics such as age, cognition or clinical status predicting outcome. ${ }^{2}$

\section{Limitations}

A clear limitation of this and many other studies examining neuroplasticity related to cognitive remediation in schizophrenia is the small sample size and limited power. Thus, it is possible that some significant relationships were not detected in our analyses. Additional studies with larger samples will be required to more clearly understand the specific effects of cognitive remediation and the neural processes that support neuroplasticity. However, studies such as this can be combined in meta-analytic investigations to test hypotheses about the impact of cognitive remediation on both cortical and subcortical brain areas in schizophrenia. ${ }^{3} \mathrm{~A}$ second methodological limitation of the current investigation is the measurement of plasticity with GLM; an approach that is only sensitive to increases and decreases in functional activation. Emerging evidence indicates that aberrant neural connections may also characterise the pathophysiology of schizophrenia, ${ }^{28}$ which these analyses cannot detect. Finally, the current analysis followed experimental rather than clinical trial conventions by including only those participants with both pre- and post-test data. We chose this approach because participant non-completion of the imaging protocol was not linked to non-completion of the treatment protocol.

\section{Implications}

This triple-blind, placebo-controlled study demonstrates that working memory-focused training has a modest influence on prefrontal plasticity in patients with schizophrenia. We observed a group $\times$ time interaction in favour of the cognitive remediation group in a subset of the left DLPFC, but were unable to replicate previously observed findings in other ROIs. Although change in DLPFC activation correlated with improvement on the N-back task, it did not correlate with improvements in external measures of working memory, attention or cognition. However, it did modestly correlate with improvement on a measure of functional capacity, suggesting that these types of interventions may influence domains outside of their direct training goals. Further examination of working memory-focused cognitive remediation will be required to definitively understand how it might influence neural activity supporting cognition and functioning more broadly. 


\section{Funding}

This research was supported by a Rehabilitation Merit Grant (D6981R) awarded by the Department of Veteran Affairs, Veterans Health Administration, Office of Research and Development and a grant from the Minnesota Veterans Medical Education and Research Foundation. Imaging was conducted at the University of Minnesota Center for Magnetic Resonance Research supported by an Institutional Center Cores for Advanced Neuroimaging Grant No: 1P30 NS076408. I.S.R. was supported by a NIMH F31 NRSA Grant No: 1F31MH106080-01. The contents of this paper do not represent the views of the Department of Veterans Affairs or the United States Government.

Ian S. Ramsay, PhD, Department of Psychology, University of Minnesota and Minneapolis VA Health Care System, Minneapolis, Minnesota; Tasha M. Nienow, $\mathrm{PhD}$, Minneapolis VA Health Care System and Department of Psychiatry, University of Minnesota, Minneapolis, Minnesota; Matthew P. Marggraf, MS, Minneapolis VA Health Care System, Minneapolis, Minnesota; Angus W. MacDonald, PhD, Departments of Psychology and Psychiatry, University of Minnesota and Minneapolis VA Health Care System, Minneapolis, Minnesota, USA

Correspondence: Angus W. MacDonald, Department of Psychology, University of Minnesota, N219 Elliot Hall 75 E. River Rd, Minneapolis, MN 55455, USA. Email: angus@umn.edu

First received 19 Jun 2015, final revision 25 Jun 2016, accepted 2 Sep 2016

\section{References}

1 McGurk SR, Twamley EW, Sitzer DI, McHugo GJ, Mueser KT. A meta-analysis of cognitive remediation in schizophrenia. Am J Psychiatry 2007; 164: 1791-802.

2 Wykes T, Huddy V, Cellard C, McGurk SR, Czobor P. A meta-analysis of cognitive remediation for schizophrenia: methodology and effect sizes. Am J Psychiatry 2011; 168: 472-85.

3 Ramsay IS, MacDonald AW. Brain correlates of cognitive remediation in schizophrenia: activation likelihood analysis shows preliminary evidence of neural target engagement. Schizophr Bull 2015; 41: 1276-84.

4 Carter CS, Perlstein W, Ganguli R, Brar J, Mintun M, Cohen JD. Functional hypofrontality and working memory dysfunction in schizophrenia. Am J Psychiatry 1998; 155: 1285-7

5 Silver H, Feldman P, Bilker W, Gur RC. Working memory deficit as a core neuropsychological dysfunction in schizophrenia. Am J Psychiatry 2003; 160 1809-16.

6 Granholm E, McQuaid JR, McClure FS, Auslander LA, Perivoliotis D, Pedrelli P, et al. A randomized, controlled trial of cognitive behavioral social skills training for middle-aged and older outpatients with chronic schizophrenia. Am J Psychiatry 2005; 162: 520-9.

7 Haut KM, Lim KO, MacDonald A. Prefrontal cortical changes following cognitive training in patients with chronic schizophrenia: effects of practice, generalization, and specificity. Neuropsychopharmacology 2010; 35: 1850-9.

8 Kurtz MM, Seltzer JC, Fujimoto M, Shagan DS, Wexler BE. Predictors of change in life skills in schizophrenia after cognitive remediation. Schizophr Res 2009; 107: 267-74.

9 Fiszdon JM, Cardenas AS, Bryson GJ, Bell MD. Predictors of remediation success on a trained memory task. J Nerv Ment Dis 2005; 193: 602-8.

10 Fiszdon JM, McClough JF, Silverstein SM, Bell MD, Jaramillo JR, Smith TE. Learning potential as a predictor of readiness for psychosocial rehabilitation in schizophrenia. Psychiatry Res 2006; 143: 159-66.
11 First MB et, Spitzer RL, Gibbon M, Williams JBW. Structured Clinical Interview for DSM-IV Axis I Disorders, Clinician Version (SCID-CV) for DSMIV. New York State Psychiatric Institute, 1997.

12 Wechsler D. Wechsler Test of Adult Reading: WTAR. Psychological Corporation, 2001.

13 Gevins AS, Cutillo BC. Neuroelectric evidence for distributed processing in human working memory. Electroenceph Clin Neurophysiol 1993; 87: 128-43.

14 Nuechterlein $\mathrm{KH}$. Vigilance in schizophrenia and related disorders. In Handbook of Schizophrenia Vol 5: Neuropsychology, Psychophysiology, and Information Processing (eds SR Steinhauer, JH Gruzelier and J Zubin): 397-433. Elsevier, 1991.

15 Nuechterlein KH, Green MF, Kern RS, Baade LE, Barch DM, Cohen JD, et al. The MATRICS consensus cognitive battery, part 1: test selection, reliability, and validity. Am J Psychiatry 2008; 165: 203-13.

16 Patterson TL, Goldman S, McKibbin CL, Hughs T, Jeste DV. UCSD Performance-Based Skills Assessment: development of a new measure of everyday functioning for severely mentally ill adults. Schizophr Bull 2001; 27 235-45.

17 Patterson TL, Moscona S, McKibbin CL, Davidson K, Jeste D V. Social skills performance assessment among older patients with schizophrenia. Schizophr Res 2001; 48: 351-60.

18 Birchwood M, Smith J, Cochrane R, Wetton S, Copestake S. The Social Functioning Scale. The development and validation of a new scale of socia adjustment for use in family intervention programmes with schizophrenic patients. Br J Psychiatry 1990; 157: 853-9.

19 Dingemans PM. Brief Psychiatric Rating Scale (BPRS). J Clin Psychol 1990; 46 $168-74$.

20 Subramaniam K, Luks TL, Fisher M, Simpson GV, Nagarajan S. Supplemental information computerized cognitive training restores neural activity within the reality monitoring network in schizophrenia. Neuron 2012; 73: 842-53.

21 Kurtz MM, Seltzer JC, Shagan DS, Thime WR, Wexler BE. Computer-assisted cognitive remediation in schizophrenia: what is the active ingredient? Schizophr Res 2007; 89: 251-60.

22 Subramaniam K, Luks TL, Garrett C, Chung C, Fisher M, Nagarajan S, et al. Intensive cognitive training in schizophrenia enhances working memory and associated prefrontal cortical efficiency in a manner that drives long-term functional gains. Neuroimage 2014; 99: 281-92.

23 Bor J, Brunelin J, d'Amato T, Costes N, Suaud-Chagny M-F, Saoud M, et al. How can cognitive remediation therapy modulate brain activations in schizophrenia? An fMRI study. Psychiatry Res 2011; 192: 160-6.

24 Minzenberg MJ, Laird AR, Thelen S, Carter CS, Glahn DC. Meta-analysis of 41 functional neuroimaging studies of executive function in schizophrenia. Arch Gen Psychiatry 2009; 66: 811-22.

25 Bowie CR, Reichenberg A, Patterson TL, Heaton RK, Harvey PD. Determinants of real-world functional performance in schizophrenia subjects: correlations with cognition, functional capacity, and symptoms. Am J Psychiatry 2006; 163: 418-25.

26 McClure MM, Bowie CR, Patterson TL, Heaton RK, Weaver $\mathrm{C}$, Anderson $\mathrm{H}$ et al. Correlations of functional capacity and neuropsychological performance in older patients with schizophrenia: evidence for specificity of relationships? Schizophr Res 2007; 89: 330-8.

27 Twamley EW, Burton CZ, Vella L. Compensatory cognitive training for psychosis: who benefits? Who stays in treatment? Schizophr Bull 2011; 37 (suppl 2): S55-62.

28 Pettersson-Yeo W, Allen P, Benetti S, McGuire P, Mechelli A. Dysconnectivity in schizophrenia: where are we now? Neurosci Biobehav Rev 2011; 35: 1110-24. 